\title{
Essai de visualisation des attitudes des principaux partis politiques belges
}

\author{
par Henri CAPRON, \\ Ingénieur Commercial, Licencié spécial en Econométrie, \\ Chargé d'enseignement l'INUFOP (FUCAM) \\ et Jean-Louis KRUSEMAN,
}

Docteur en Droit, M.A. in Economics, Chargé de cours a la FUCAM,

Chargé d'ensefgnement à l'INUFOP (FUCAM).

\section{Introduction.}

L'pbjectif poursuivi par cette recherche est d'offrir une vision la plus précise possible de la scène politique nationale. Dans le cadre de ce travail, nous avons été amenés à adopter une méthode d'analyse statistique conduisant à une synthèse du tableau original des données en en faisant ressortir les composantes essentielles des informations qui y sont contenues.

Pour ce faire, nous tenterons dans les sections qui suivent de dégager une image globale et synthétique des positions des principaux partis politiques belges. Après un bref rappel du contexte politique (section 2), nous expliciterons à l'aide de l'analyse factorielle les attitudes politiques caractérisant les principaux partis politiques belges à propos des questions les plus importantes débattues à la veille des élections du 17 décembre 1978 (section 3). Nous avons estimé qu'il serait intéressant également de relever, dans une quatrième partie, les éventuelles modifications d'attitudes - avec quelque prudence étant donné les différences de contenu - de ces partis entre 1974 et 1978, en nous référant à l'analyse des programmes politiques défendus en 1974, réalisée par A. P. Frognier, nous concluerons ensuite en suggérant quelques pistes de recherches futures. 


\section{Evolution des partis politiques belges depuis 1946 .}

Les observateurs de la scène politique belge actuelle semblent d'accord pour distinguer trois grandes catégories de clivages (1). Bien que, tour à tour, les problèmes confessionnels (opposant les catholiques aux autres familles politiques) et socio-économiques (déterminant un clivage "gauche-droite ») ont prédominé entre 1930 et 1958, l'élément linguistique émergea comme nouveau facteur distinctif essentiel après 1958.

Ce clivage linguistique entre communautés d'expression française et d'expression néerlandaise a relégué au second plan un autre élément distinctif caractérisant chacune des communautés linguistiques. Il s'agit d'un clivage au second degré entre partisans de l'unitarisme et les tenants de l'option fédéraliste. En réalité, comme l'énonce Rowies (2), quoique fédéralistes et unitaristes réagissent différemment aux problèmes intercommunautaires, ceci ne signifie nullement que les unitaristes de part et d'autre de la frontière linguistique témoignent d'une identité de vue, ni que les fédéralistes flamands partagent les mêmes idées que leurs homologues wallons. La conception d'un type unitariste ou de type fédéraliste pouvant éventuellement être assez différente selon la communauté considérée.

Si les élections de 1968 ont été considérées comme le triomphe de l'« extrémisme linguistique » (3), il apparaît maintenant que les partis linguistiques n'ont pas réussi à conserver leur emprise sur l'électorat et ont perdu du terrain aux élections de 1974 et de 1978.

Le linguistique ne semble plus être le thème politique dominant qu'il fut en 1968. La lutte contre le chômage, affectant autant le nord que le sud du pays, le soutien aux secteurs en difficulté, la nature des compétences dont se départira l'administration centrale au profit des autorités régionales et communautaires, telles sont les questions cruciales, agrémentées le cas échéant de considérations linguistiques, qui prédominent

(1) Une bonne synthèse de l'évolution du système politique en Belgique est présentée, notamment par RABUSHKA et SHEPSLE (1972), Politics in Plural Socheties: A Theory of Democratic Instability, Merrill Political Science serles et ROWIES (1975), «Les partis polftiques en Belgique», Dossiers du CRISP, Brussels. En ce qui concerne le phénomene des clivages proprement dit en Belgique, on peut se reporter a J. LADRIERE, «Le système politique belge : situation 1970 in Courrier hebdomadaire du CRISP, n' 500, 20 novembre 1970. Rappelons que les clivages en Wallonie ont fait l'objet d'une étude par FROGNIER et SEILER, \& Les Clivages en Wallonie, une application à l'analyse factorielle \$, Courrier hebdomadaire du CRISP, n० 583, 14 décembre 1972, (1972).

(2) Op. cit., p. 7.

(3) RABUSHKA et SHEPSLE, op. oit., p. 117. 
ces dernières années (4). C'est dans ce contexte de crise économique et de chômage croissant qu'interviennent les élections de décembre 1978. La présentation des attitudes des partis à cette occasion fait l'objet du point suivant.

\section{Une typologie des attitudes politiques adoptées par les principaux partis politiques en décembre 1978.}

\subsection{Les données.}

Déclenchées par la démission du gouvernement présidé par Léo Tindemans (dirigeant une équipe soutenue par une coalition des partis PSC et CVP, PSB et BSP, VU et FDF), de nouvelles élections furent organisées le 17 décembre 1978. A la veille de ces élections, les opinions des principaux dirigeants de partis ont été recueillies à l'occasion de divers entretiens portant sur leurs attitudes respectives à l'égard des problèmes de nature socio-économique et institutionnelle auxquels le pays est confronté.

Les données utilisées dans cette section sont tirées de deux séries d'interviews publiées respectivement par les quotidiens Le Soir et l'Echo de la Bourse (5).

La première source d'information traite exclusivement de questions institutionnelles. Nous avons regroupé celles-ci en sept thèmes principaux (voif annexe I). La mise en œuvre des réformes institutionnelles et la valeur attribuée au pacte d'« Egmont » en sont les deux premiers. Les deux suivants traitent des compétences respectives des régions et des qommunautés. La protection des minorités et la répartition linguistique du pouvoir décisionnel en constituent deux autres, le dernier étant réserté au statut de Bruxelles et des communes périphériques. Le second ensepple de données concerne, tout à la fois, les questions de nature institutionnelle et socio-économique. Nous les avons structurées en axes distinctifs des programmes de partis répartis en trois groupes : les caractéristiques des programmes eux-mêmes, les questions institutionnelles et les problèmes économiques (voir annexe II).

Les deux séries de réponses ont été traitées selon des approches distinctes :

(4) Il convlent de noter cependant que RABUSHKA et SHEPSLE prennent en compte également les crises économlques graves en tant qu'évènements exogènes affectalt la politique communautafre et modiflant l'évolution politique de soclétés plurale (op. cit., p. 92).

(5) Io Botr, 14, 15 et 16 décembre 1978, et l'Echo de la Bourse, 23, 24-26, 27 et 29 novenbre, 4, 7, 11, 13 et 14 décembre 1978. 
1. En ce qui concerne les réponses parues dans Le Soir, nous avons utilisé une procédure de description logique sous forme disjonctive complète. A partir de l'ensemble de questions (Q), nous avons construit pour chaque question spécifique (q) un sous-ensemble de cinq attitudes possibles $\left(\mathrm{q}++, \mathrm{q}+, \mathrm{q}-, \mathrm{q}--, \mathrm{q}^{\circ}\right)$. Si une réponse $\mathrm{i}$ est fournie à toute question $q$ par l'individu $j$, il est attribué un poids $k(i, j)=1$ et, dans tout autre cas, $i^{\prime} \in q, k\left(i^{\prime}, j\right)=O$. Les cinq attitudes correspondent aux types de réponses suivants : positive inconditionnelle $(\mathrm{q}++)$ ou conditionnelle $(q+)$, négative inconditionnelle ( $\left.q_{-}^{-}\right)$ou conditionnelle ( $\mathrm{q}-$ ) ou abstention $\left(\mathrm{q}^{\circ}\right)$. La dernière attitude recouvre le fait que le sujet n'a pas donné de réponse au thème abordé ou n'a pas exprimé clairement son opinion sur la question. Elle porte ainsi non seulement sur les cas de non-réponse mais également sur ceux de reponses floues ou incertaines. De cette façon, recourant à cette procédure, un tableau des données originales a été traduit en une forme complètement disjonctive se résumant comme suit :

$$
\forall_{j} \varepsilon \mathrm{P}, \forall_{\mathrm{q}} \varepsilon \mathrm{Q}, \Xi_{\mathrm{i}} \varepsilon \mathrm{Q} \rightarrow\left[(\mathrm{K}(\mathrm{i}, \mathrm{j})=1) \Lambda\left(\mathrm{i}^{\prime} \varepsilon \mathrm{q}, \mathrm{i}^{\prime} \neq \mathrm{i}\right) \rightarrow \mathrm{K}\left(\mathrm{i}^{\prime} \mathrm{j}^{\mathrm{j}}\right)=0\right]
$$

où $\mathrm{P}$ représente l'ensemble des partis et $\mathrm{Q}$ l'ensemble des questions à plusieurs modalités de réponse I. Bien que ceci caractérise la démarche initialement adoptée, les résultats présentés ci-après sont issus d'une analyse portant sur un tableau transformé des données permettant une lecture plus simple des résultats.

2. Les réponses reproduites par l'Echo de la Bourse ont également été transposées sous la forme d'un tableau de description logique sous forme disjonctive mais en adoptant une procédure de décodage sensiblement différente de la précédente. Dans l'approche précédente, les questions ont en fait été décomposées en un ensemble de thèmes pour lesquels on a relevé les attitudes caractérisant les partis. Ici, l'hététogénéité des questions, tantôt ' conjoncturelles ' (par exemple, les chances de réussite de la prochaine constituante), tantôt ponctuelles (par exemple, la suppression de la taxation des investissements) nous a conduit à adopter un principe de codage différent et à procéder à une double analyse, la première sur l'ensemble des questions, la seconde sur les questions plus ponctuelles. D'autre part, contrairement au premier questionnaire, par lequel les partis se prononcent pour ou contre un certain nombre de points spécifiques, ce questionnaire oblige les partis à révéler leurs préférences et à ébaucher la solution qu'ils proposent à l'égard des problèmes les plus importants. Ainsi, les partis se distinguent suivant l'option choisie pour solutionner les problèmes soulevés. Dans l'ensemble, les options sont contradictoires mais certaines dénotent simplement une perception différente du problème. 
Préalablement à la présentation des résultats de l'analyse, nous avons jugé utile de reprendre une analyse effectuée par A. P. Frognier et R. Delfosse qui étudie les positions prises par les partis sur un certain nombre de problèmes de nature politique lors des élections de mars 1974. La méthodologie adoptée et la composition des données ne seront pas abordées ici et nous convions le lecteur à se référer à l'article original (6). Signalons néanmoins que leur démarche offre quelques analogieł avec celle que nous avons retenue pour la seconde série de réponses, à ceci près que leur analyse de contenu se base sur des concepts d'analyse sémiotique tandis que notre analyse se veut plus ponctuelle afin de montrer l'intérêt de la visualisation synthétique d'une problématique complexe.

\subsection{Méthodologie.}

La nature booléenne des tableaux (7) constitués et l'équivalence dans les résultats de son analyse factorielle avec ceux issus d'un tableau de contingence nous a conduit à opter pour cette méthode (8). L'ensemble des techniques reprises sous cette dénomination ont pour objet de fournir des représentations synthétiques des données initiales, tout en respectant au mieux leur contenu informatif par la détermination des facteurs dominants prenant en compte et incorporant un maximum de variabilité. Géométriquement, cette méthode consiste en une projection des observations appartenant à un hyper-espace dont les dimensions sont définies par le nombre de variables dans un sous-espace (ou espace réduit de dimension inférieure au nombre minimal d'observations et de variables) tout en déformant le moins possible le nuage de points initial (c'est-à-dire avec le minimum de perte d'information).

Les nouveaux axes ainsi dégagés et dont il convient de trouver la signification portent dans un ordre décroissant le maximum d'information et sont orthogonaux (indépendants) les uns par rapport aux autres. Chaque variable (dans notre cas, les partis) est représentée par un point dans cette nouvelle configuration spatiale extraite des données initiales. La proximité de certains points-variables et leur distance par rapport aux autres ainsi que leur dispersion aident à l'interprétation des axes.

(6) FROGNIER A.P. et DELFOSSE P. (1974), \& Le système des partis en Belgique », Res Publica, no 3-4, pp. 405-424.

(7) La simplification, discutée ci-après, Introduite dans le tableau lssu des donnés Le Soir par la suppression des attitudes < conditionnelles * et leur remplacement par des nombres fractionnalres constitue une entorse $\$$ possible a la forme booléenne sans Incidences notables sur les résultats de l'analyse.

(8) Sur ce polnt précls, volr notamment BENZECRI J.P. (1973), L'analyse des donnes; Tome II : l'analyse des correspondances, Dunod, Paris. 
En effet, deux variables seront d'autant plus liées, corrélées, que les points-variables seront proches entre eux et éloignés de l'origine. Le choix de la mêthode a été conditionné par la spécificité de nos statistiques et les objectifs de l'étude. La présentation particulière des données, sous forme de tableaux de description logique nous a conduit à recourir à l'analyse factorielle des correspondances qui s'avère bien appropriée au traitement de tels tableaux (9). Nous sommes, de plus, intéressés par le positionnement relatif non seulement des partis, mais également des questions, ce qui permettra de visualiser les questions (ou types de problèmes) qui démarquent le plus les partis et, par ailleurs, de faciliter l'interprétation des axes. Ce type d'analyse se présente en fait comme une double analyse en composantes principales (en partant, pour la première, des partis et, pour la seconde, des questions) d'un espace muni d'une métrique particulière, la distance du $\mathrm{x}^{2}$. Pour l'application de nos données, nous nous limiterons à la présentation des trois premiers axes illustrant les associations les plus représentatives entre les facteurs et les variables.

Signalons, pour terminer, que si la facilité qu'apporte la méthode d'analyse par projection des données dans des espaces préalablement réduits, est réelle du point de vue de la visualisation des observations dans l'espace, il reste que l'interprétation des axes factoriels en termes de variables latentes n'a qu'une valeur d'hypothèse explicative. $\mathrm{Si}$, comme le souligne très bien Guigou (10) les tableaux analysés ne sont pas reptésentatifs d'un système cohérent, l'observateur sera fort en peine de proposer une interprétation des axes factoriels et à retenir des hypothèses caractérisant l'ensemble d'observations analysé. Autre conséquence de ce type d'analyse, la qualité de l'interprétation des relations entre les différents éléments, observations et variables, est directement fonction du soin apporté au choix et à la réalisation du codage de l'information, d'une part, et de la sélection sensée des points-variables et des points-observations contribuant de la manière la plus statistiquement significative à la constitution d'un facteur, d'autre part.

\subsection{Résultats.}

3.3.1. Analyse des clivages linguistique et régional (1978).

Préalablement à la présentation des résultats et à leur discussion, il nous parait utile de commenter brièvement diverses approches investi-

(9) Pour une présentation formelle de la méthode, voir BENZECRI, op. cit. Pour une présentation plus synthétique, voir GIGOU J.L. (1977), Methodes multdimensionnelles, Analyse des donnes et Choix a criteres multiples, Dunod, Paris. (10) Op. alt., p. 131. 
guées. L'analyse des données présentées ci-dessus, sous forme disjonctive complète, a été reprise sous diverses formes donnant des résultats sensiblement similaires. Les différences entre ces approches portent sur la codification des réponses conditionnelles et de l'abstention. Les divers types de codage testés ainsi que leur répartition dans le tableau de données sont repris en annexe III. Le codage de type I - qui correspond à celui décrit précédemment - reconnaît à chaque attitude une spécificité propre. Celui-ci n'a pas été retenu car l'analyse du tableau des données fait ressortir un nombre relativement faible de réponses conditionnelles. Le second codage répartit les réponses conditionnelles parmi les inconditionnelles en leur accordant un poids de $1 / 2$. Le troisième type de codage ne concerne que la prise en considération des réponses suivant qu'elles dénotent une attitude positive ou négative, sans prendre en compte le phénomène d'bstention. Enfin, le codage IV est celui que nous avons retenu. En fait, il est identique au codage II mais ne considère pas l'abstention. Ce defnier a été retenu car ses résultats ne diffèrent pas de ceux obtenus pour les autres et permet une présentation plus simple des résultats. De l'analyse du tableau de données et de leur corrélation, un certain nombre de constatations peuvent être relevées.

Les sommes marginales des colonnes qui représentent le nombre de réponses émises par les divers partis sont représentées par l'histogramme citdessous. La lecture de ce graphique montre que le PC a émis le plus de réponses clairement interprétables. D'autre part, les partis francophones (sauf le RW) ont un taux de réponses supérieur à celui observé pour les partis néerlandophones (11). Cela signifie que les partis francophones ont une tendance à exprimer leur position plus clairement que les partis néerlandophones. D'une manière générale, on remarquera le taux élevé d'abstentions - environ $34 \%$ - ce qui manifeste de la part des partis, une tendance à éluder les questions embarrassantes.

Si nous passons maintenant à l'analyse de la matrice de corrélation entre partis suite aux réponses émises, nous sommes en mesure d'apprécier le degré d'associativité entre les divers partis. Sur base de cette matrice de corrélation, nous avons construit un graphique (fig. 2 A) visualisant les degrés d'associativité positive les plus prononcés (degré de convergence dans les opinions émises) et un second (fig, 2 B) reprenant les degrés d'as-

1) Rappelons que ne sont enregtstrées que les réponses clairement exprimées. Solt considérées comme non-réponses les réponses ambiguěs (c'est-a-dire telles qu'on ne peut distinguer sl le parti considéré est effectivement pour ou contre le probleme souleve) et la discussion de problemes n'ayant rien a voir avec celui qui est posé. 


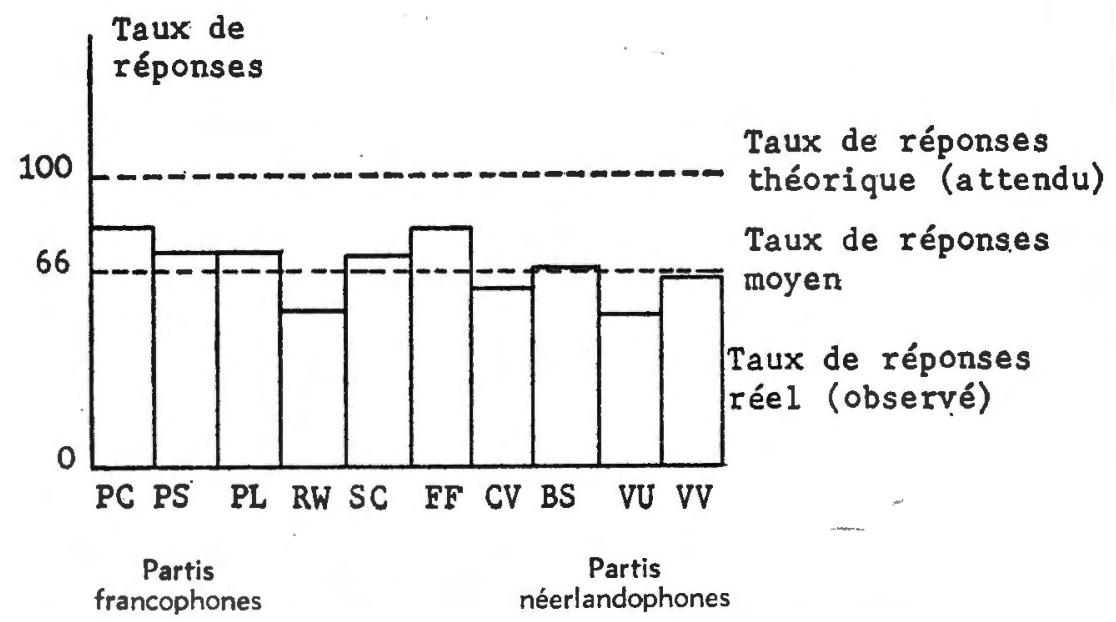

FIG. 1. - Taux de réponses aux questions linguistiques.

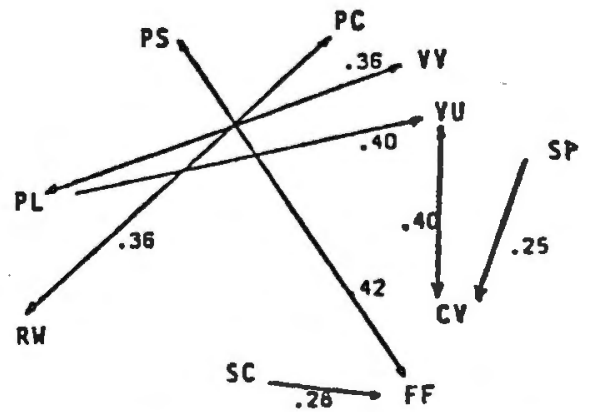

FIG. 2 A. - Graphe des associativités positives les plus fortes entre partis pour les questions linguistiques.

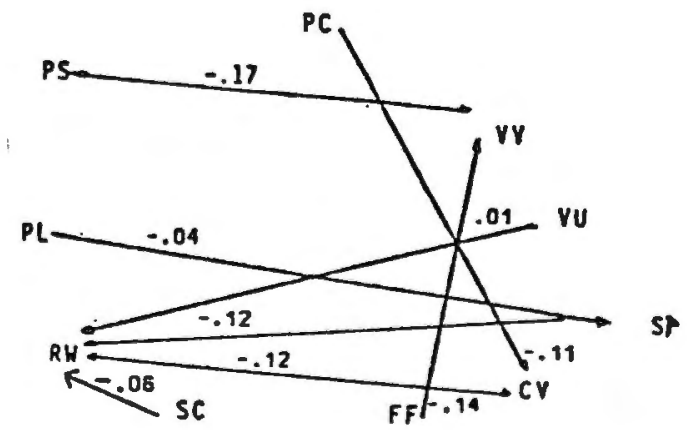

FIG, 2 B. - Graphe des associativités négatives les plus fortes (ou positives les plus falbles) entre partis pour les questions linguistiques. 
sociativité négative les plus forts ou les degrés d'associativité positive les plus faibles (degré de divergence dans les opinions émises) (12).

Comme nous pouvions nous y attendre, le premier graphe rassemble, d'un côté, les partis francophones et, de l'autre, les partis néerlandophones. Au niveau des familles politiques, seule la famille libérale conserve un certain degré d'homogénéité dans ses prises de position en la matière. En effet, les prises de position du PVV sont le plus liées à celles du PL et inversement (du moins au second rang, après la VU). Par contre, le second graphe illustre clairement les divergences entre les positions francophones et péerlandophones dont la position la plus caractéristique est celle du RW confronté à une opposition de points de vue des partis néerlandophqnes (sauf le PVV) et également d'un parti francophone, le PSC (13).

Nous verrons ci-après que ces divergences caractérisent le facteur le plup dominant issu de l'analyse factorielle des données.

Gette dernière nous a fourni cinq valeurs propres utiles expliquant $72,7 \%$ de l'inertie totale. Nous n'analyserons de manière détaillée que les trois premiers axes et ne mentionnerons que ce qui les caractérise pour les deux derniers. Les trois premiers expliquent respectivement $21,2 \%$, $16,4 \%$ et $14,3 \%$ de l'inertie, soit un total de $51,9 \%$. Pour clarifier l'exposé ,nous ne prenons en considération que les observations dont les corfélations sont fortes avec chacun des facteurs et faibles avec les autres. La feprésentation graphique des variables et des observations explicatives pou chacun des trois facteurs retenus est illustrée aux figures 3 et 4 . Le prequier facteur op̀re une séparation parfaite entre les partis francophones et néerlandophones. Facteur de niveau général, il exprime l'opposition linguistique et l'intensité de la divergence d'opinions suscitée par les proplèmes qui en découlent. L'opposition la plus forte se manifeste entre le RW et le CVP tandis que le PSC se situe sur une position intermédiaire entre les autres partis francophones et les partis néerlandophones. Le second facteur illustre les divergences de vues entre socialistes d'une part, et le PVV d'autre part, en ce qui concerne l'acceptation du pacte d'Egmont et la distribution des responsabilités décisionnelles. On constate que les partis francophones se montrent, à l'exception du RW, favorables au pacte d'Egmont, tandis que les partis néerlandophones - pour être allés trop loin dans les concessions - paraissent le réfuter (à l'exception du

(12) Nous tenons a souligner que cette démarche ne constitue en rien une analyse de causalitê mals blen une analyse d'associativitê conformément à l'interprétation des coefficients de corrélation représentant des mesures d'association.

(13) Notons que pour des donnés binalres, un coefficient de corrélation nul entre deux séries correspond à une convergence (divergence) de points de vue de $50 \%$. 


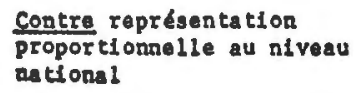

(207)

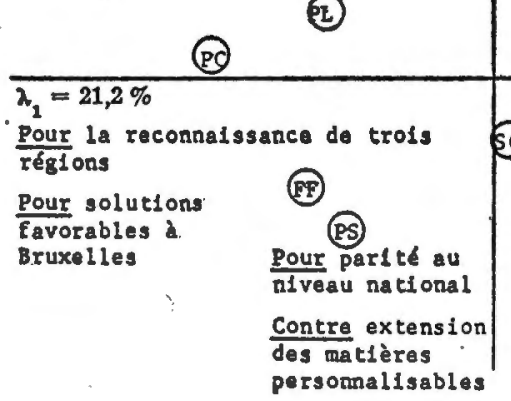

Pour extension

des matieres

personalisebles

FIG. 3. - Problèmes linguistiques et régionaux (1978). Représentation des axes factoriels 1 et $\mathbf{2}$

Contre Interdépendance du

communatalre ot du socio-économlque

Contre délimitation

nouvelle de

Bruxelles

Contre protection

Si des minorités

périphér1e

bruxallolse

(2) 
BSP). L'axe 3 oppose principalement le RW et le BSP au PSC pour les thèmes portant sur l'application des réformes institutionnelles, la protection des minorités et le problème de Bruxelles. Le quatrième axe montre l'opposition entre le CVP, la VU et le PSB, d'une part, et le PSC, le BSP et le PVV, d'autre part, en matière de compétences régionales et communautaires. Il est symptomatique de retrouver d'un côté les deux partis régionaux dominants (CVP et PSB) et, de l'autre, les partis appartenant aux mêmes familles, mais minorisés dans leurs régions respectives (PSC, BSP). Le cinquième axe est spécifique au $\mathrm{PC}$ qui se différencie des autres partis et particulièrement du RW et du PSC (14) notamment par son attitude négative à l'égard du pacte d'Egmont, de la résolution des conflits de compétence et de la fixation des limites des régions politiques.

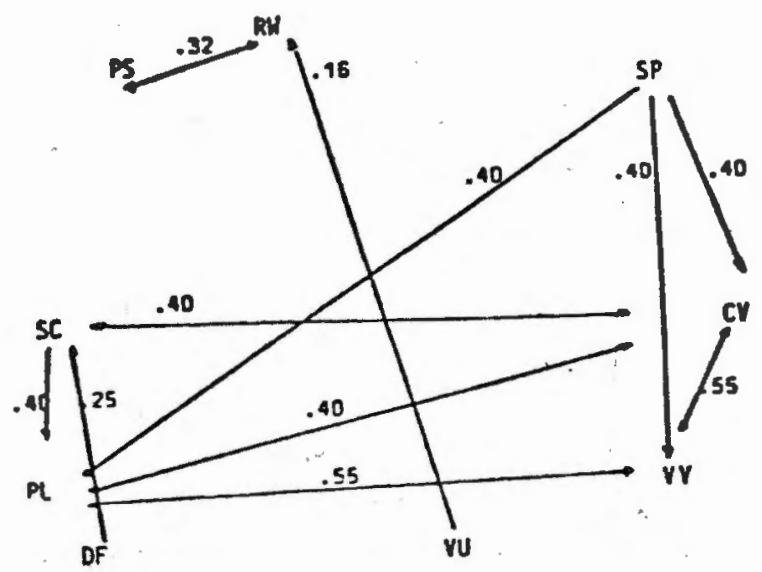

FIG. 5 A. - Graphe des associativités positives les plus fortes entre partis pour les thèmes abordés en 1978.

3.3.2. Analyse des programmes des partis en 1978 .

Comme pour l'analyse précédente, il nous paraît intéressant de visualiser sur un graphe les corrélations marquant les convergences et les divergences les plus fortes entre les partis. Les graphes issus de la matrice de corrélation sont présentés à la figure 5 . Les partis néerlandophones se caractérisent par une plus grande homogénéité de réponses (mis à part le VU) que les partis francophones. Comme précédemment, le RW semble se démarquer des autres partis, non seulement des socio-chrétiens mais également des libéraux. Les partis peuvent être rassemblés en deux blocs :

(14) Cet axe explique $10 \%$ de l'inertle totale. 
le PS, le RW et la VU qui sont unis par une seule relation et les autres partis entre lesquels on observe une plus grande homogénéité d'opinions. Dans ce dernier groupe, le FDF constitue un parti marginal dans la mesure où la relation l'unissant aux autres est de faible ampleur. Ces premières constatations sont confirmées par l'analyse factotielle des données.

Cette dernière nous a fourni quatre valeurs propres utiles nous expliquant $83,7 \%$ de l'inertie totale. La représentation graphique des trois premiers axes factoriels est donnée aux figures 6 et 7 . Les trois premiers axes interviennent pour, respectivement, $32,2 \%, 23,2 \%$ et $14,7 \%$ dans la répartition de l'inertie totale. Le premier axe place d'un côté les partis communautaires (FDF, VU et RW) et le PSB en opposition aux autres partis. Nous pouvons le considérer comme représentatif du degré d'auto-

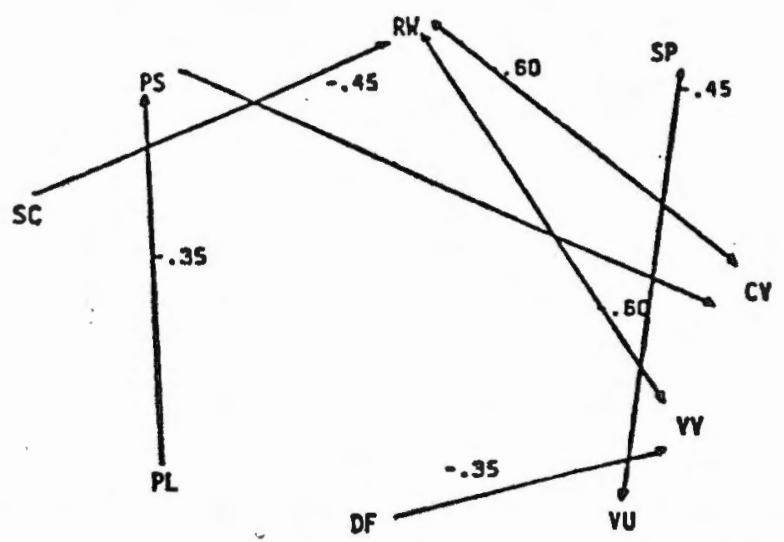

FIG. 5 B. - Graphe des assoclativités négatives les plus fortes entre partis pour les thèmes abordés en 1978.

nomie socio-économique revendiquée, avec le PSB et le RW optant pour une autonomie large doublée d'un interventionnisme public très prononcé et les familles libérales et socio-chrétiennes se montrant favorables à une autonomie restreinte et à un degré d'interventionnisme public limité. Sur l'axe 2, on observe une opposition des thèses fédéralistes (VU et FDF) où le dogmatisme des partis d'opposition extrémistes s'oppose au réalisme des partis de gouvernement en ce qui concerne le déficit budgétaire, l'assainissement des finances publiques et le type d'intervention.

Un résultat sensiblement identique est obtenu lorsqu'on recommence l'analyse en enlevant des données les questions qui traitent du programme des partis et des problèmes institutionnels. La figure 8 illustre le changement d'axes et leur rotation provoqués par cette nouvelle analyse comparativement à ceux obtenus lorsqu'on prend en considération l'en- 


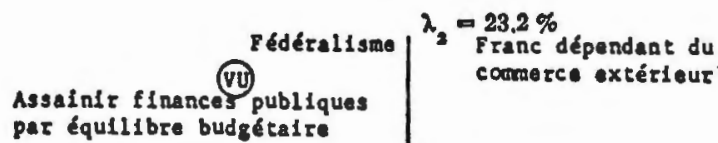

Primuté a l'fostet tutionnel

Maltzise du deflett flin. pub. aprete reformes institutioanelles

(6)

Autonomic en nombreuses matidres

Intervention de

I'Etat sur les

structures

Indus trielles

Développenant de

l'Initiatieve publtque

Interventsons

directues to

étendus de l'ztat

(e)

Protection du franc

contre ta spéculation

Résorption du déficlt $f\lfloor n$. pub.

par politique fin. \& soc. réliste

FIG. 6 - Programmes des partis en 1978. Représentation des axes factoriels 1 et 2.

\section{(2D) (1) $_{\lambda_{1}}=32,2 \%$}

Autonomle régio-

aule restreinte

(3)

Inturventions

gouvernementales

SP ponctuelles

Problèmes socio-écononlques

sont prépondérants

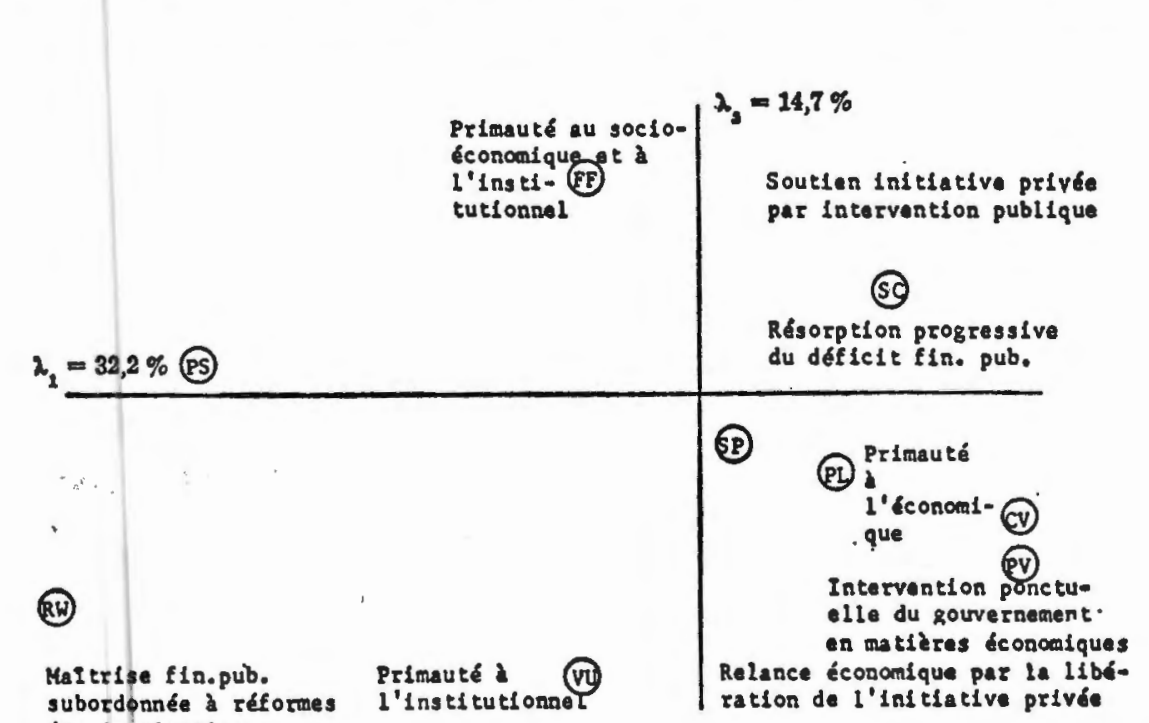

inatitutionot

ration de l'inl tiative privíe

FIG. 7. - Programmes des partls en 1978. Représentation dea axes factoriels 1 et 3. 


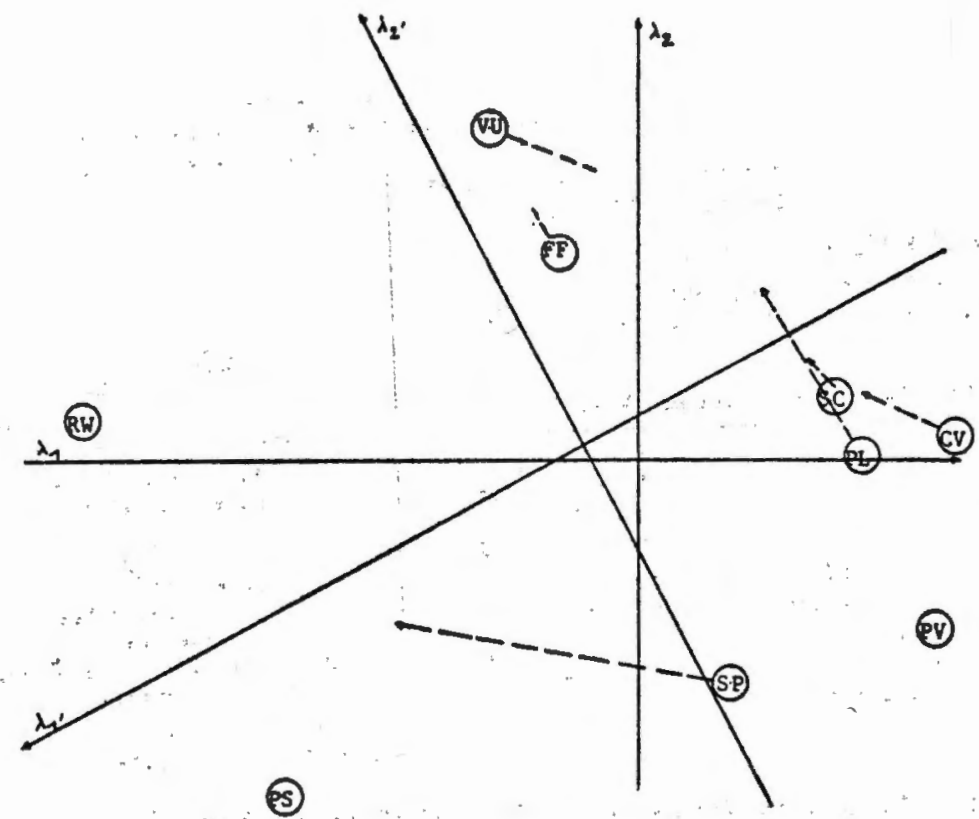

FIG, 8. - Programme des partis (1978).

Représentation de la rotation des axes factorlels 1 et 2.

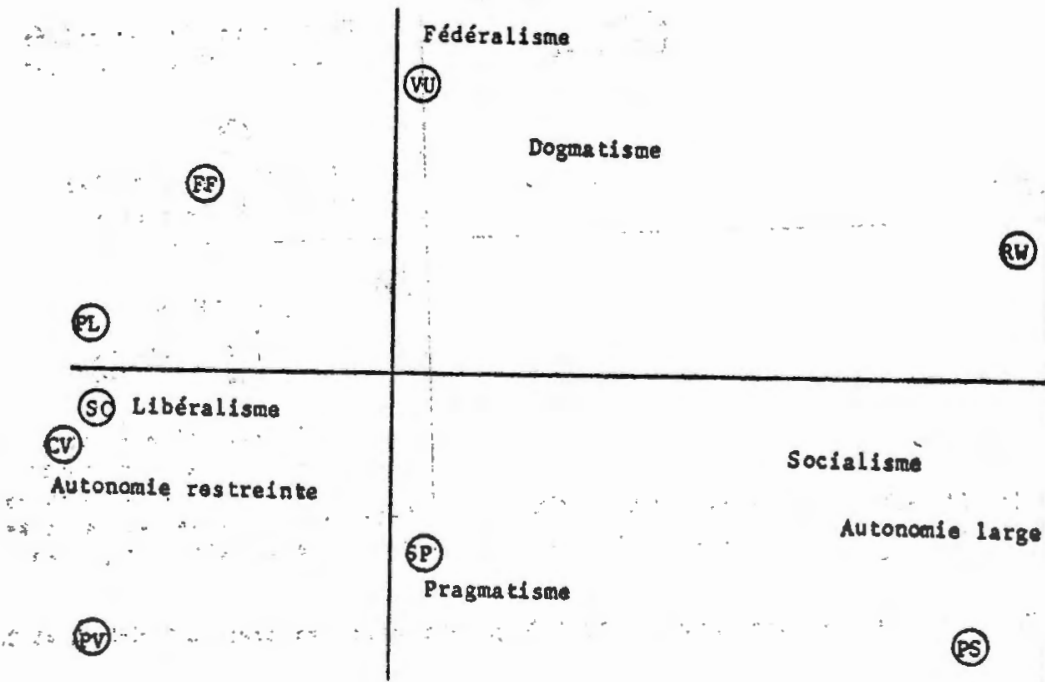

HTG. 9. - Programme des partis (1978).

Représentation synthétique des attitudes des partis. 
semble des questions. On observe en traits discontinus le déplacement dans la position des partis suite à la seconde analyse. Le BSP réalise le déplacement le plus notable par un rapprochement de la position du PSB tandis que le PL s'écarte fortement vers celle occupée par le: PVV. Iee retrait des questions traitant des problèmes institutionnels qui écartaient le BSP du PSB dans la première analyse a permis ce rapprochement. La distanciation du PL par rapport au PVV se marque par une tendance plus prononcée du PL au dogmatisme par rapport au réalisme du PVV. L'examen de l'ensemble du plan des deux premiers facteurs conduit à diviser les partis en quatre groupes en foriction du quadrant dans lequel ils sont situés. D'une part, nous avons les socialistes et le RW qui se démarquent par leur préférence pour le socialisme et le large degré d'autonomie revendiquée mais se distinguent entre eux par le dogmatisme du RW s'opposant au pragmatisme des socialistes. D'autre part, nous avons l'ensemble des autres partis, d'option plus libérale mais se départageant en fédéraliste et dogmatique (FDF et VU) d'un côté, et réaliste et partisan de l'autonomie restreinte, de l'autre (SC, CV, PV et, dans une moindre mesure, PS et PL). Ceci est synthétisé à la figure 9 (15). Le troisième axe met en opposition le PSC et le FDF au VU, ce dernier accordant une plus grande confiance dans l'initiative privée. L'axe 4 - non repris ici caractérise les divergences de vue au' sein de la famille socialiste (BSP et $\mathrm{PSB}$ ) en matière de programme et sur certaines questions de nature économique.

\section{Analyse statique comparée des convergences et des divergences dans les attitudes politiques observées en 1974 et 1978.}

Dans le but d'examiner l'évolution des attitudes des partis à la suite du développement de la crise économiquie qui a ébranlé durement de larges pans de notre structure industrielle et a provoqué un accroissement inquiétant du chômage, nous nous proposons - en premier lieu - d'appliquer notre approche analytique aux donnés utilisés par Frognièr et Delfosse dans leur étude des programmes électoraux de 1974 et d'en présenter les résultats avant de tenter de comparer les attitudes observées respectivement en 1974 et 1978.

(15) Co graphique se base unlquement sur les questions traltant des prablemes Institutionnels et socio-6conamiques. 
4.1. Comparaison des attitudes politiques observées en 1974 et 1978.

Dans leur analyse, Frognier et Delfosse ont réparti les réponses des partis au questionnaire qui leur a été administré en 1974, selon des axes sémantiques prédéfinis et ont subdivisé les données en trois sous-groupes - standards moraux (16), problèmes institutionnels et socio-économiques. Nous avons opté pour l'utilisation du codage binaire qu'ils ont euxmêmes employé (17).

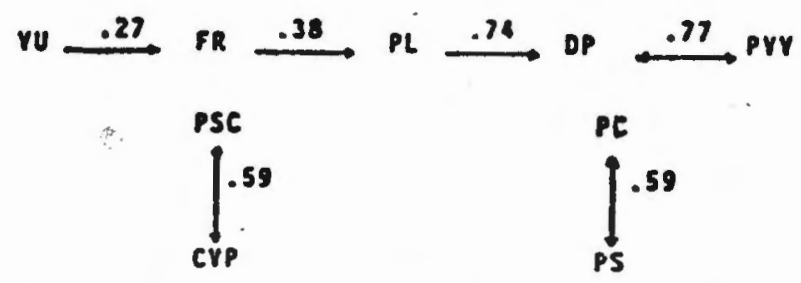

FIG. 10 A. - Graphe des associativités positives les plus fortes entro partis pour les programmes de 1974.

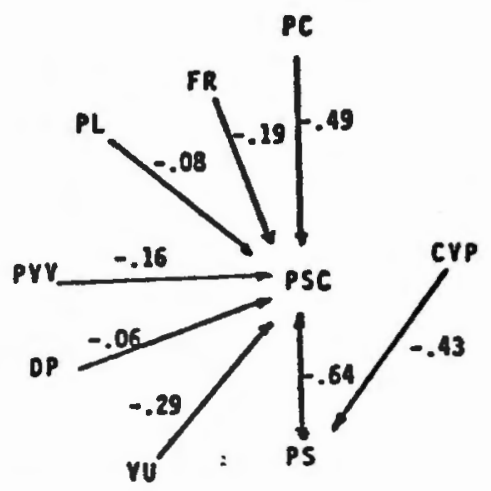

FIG. 10 B. - Graphe des associativités négatives les plus fortes entre partis pour les programmes de 1974.

Les graphes synthétiques des associations les plus fortes et les plus faibles basés sur l'analyse de corrélation et illustrés à la fig. 10, conduisent a certaines constatations intéressantes. Si nous reprenons les associations les plus fortes, on observe que les partis se divisent en trois blocs repre-

(16) Il convient de signaler que, par manque d'informations, tous les partis n'ont pu etre positionnés pour l'ensemble des questions traitant des standards moraux. C'est le cas pour le PC, le PVV et le PLDP. Ceci inclte la prudence lors des commentajres des résultats en cette matiere concernant ces partis.

(17) EROGNIER A.P. et DRLFOSSE P., op. cit., tableau III, p. 120. 
sentant chacune des grandes familles politiques auxquelles se relient les partis communautaires mais avec un moindre degré d'association. Nous observons ainsi, d'une part, les socio-chrétiens, d'autre part, les socialistes et les communistes et, dans un troisième bloc, la famille libérale et les partis communautaires. L'association entre ces deux derniers provient, d'une part, de la position intermédiaire des partis communautaires sur les matières économiques et éthiques et, d'autre part, d'une plus grande similitude d'opinions entre ces deux groupes de partis en matière régionale.

Le graphe des associations négatives les plus fortes caractérise la grande différenciation des thèses défendues par les socio-chrétiens et particulièrement le PSC comparativement aux autres partis. On constate en effet que c'est à l'égard du PSC que tous les partis s'opposent le plus intensément (mais à des degrés différents). A l'inverse, comme nous pouvions nous y attendre, l'antagonisme le plus marqué se manifeste entre les deux grandes familles politiques, les socio-chrétiens et les socialistes.

En conclusion, on peut considérer que ces graphes constituent une bonne image de la société politique belge divisée en quatre familles politiques (socio-chrétiens, libéraux, socialistes et communautaires) dans laquelle les conflits les plus importants opposent les socio-chrétiens aux socialistes et dans laquelle également on observe que le PSC traverse une crise profonde qu'il ne parvient pas à surmonter (contrairement au CVP), ce qui constitue une cible idéale pour les autres partis.

\subsubsection{Analyse par thème des observations pour 1974.}

Lłs données étant constituées en trois sous-groupes, nous avons procédé pour chacun d'eux à une analyse dont la représentation graphique des résultats est présentée à la figure 11. A la lecture de ces graphiques, on observe que les divers clivages typifiant la situation politique belge y sont clairement représentés. Les questions de nature socio-économique voient s'opposer les différentes conceptions de l'économie parmi lesquelles les partis communautaires occupent une position intermédiaire. La configuration des partis telle qu'elle ressort de l'analyse des attitudes en matière éthique visualise bien la réalité politique en opposant les socio-chrétiens aux autres partis traditionnels et ceci, bien que le parti communiste et les factions libérales ne soient positionnés que pour un nombre restreint de questions (18). Ici encore, les factions communautaires occupent une position inter-

(18) Le second axe caracterise cette particularite des données ou le PC s'oppose aux PVV et PLDP simplement parce qu'lls ne répondent qu'a đes questions différentes. Remarquons cependant que ce second axe oppose conception soclaliste et conception libérale en la matière. 
médiaire. Le dernier sous-groupe traite des problèmes institutionnels. Il est intéressant de noter, par le biais du premier axe factoriel, la fermeté de la position du PSC (concentrant quelque $83 \%$ de l'inertie totale de cet axe) en opposition à celle du PSB concernant la délégation de pouvoirs du gouvernement central vers les administrations régionales. En 1974, le PSC insiste toujours pour préserver les pouvoirs conférés aux provinces. Le second axe semble se rapporter aux modalités de l'exercice du pouvoir vers lesquelles les partis portent leurs préférences. Les partis représentant les intérêts des minorités (PLP de Bruxelles et le FDF +RW (= FR) montrant un penchant pour la démocratie directe à l'opposé des grands partis traditionnels (CVP, PSB) défendant jalousement leur pouvoir discrétionnaire de porte-parole de la volonté populaire (c'est-d̀-dire la particratie). Pour terminer ce point, il convient d'énoncer une remarque générale concernant ces analyses partielles. Il est, en effet, fort possible que le faible pouvoir explicatif de certaines proximités, de même que l'apparente contradiction révélée par certaines attitudes de partis puissent résulter : $1^{\circ}$ du traitement par trop élémentaire des données lorsqu'on attribue un poids nul à la variable quand l'information fait défaut et $2^{\circ}$ de manière plus générale, de l'étroitesse de l'échantillon relatif à chaque sous-groupe.

\subsubsection{Analyse de l'ensemble des observations pour 1974.}

La seconde étape, comme indiqué plus haut, a été de procéder à l'analyse de l'ensemble des observations concernant tous les partis. Frognier et Delfosse ont procédé, dans ce cas, à une analyse en facteurs communs qui les a conduits à interpréter leur premier axe comme indiquant la position des partis selon leur degré d'hétérogénéité idéologique. Le deuxième axe dans leur analyse représente l'axe sémantique qu'ils ont adopté a priori, c'est-à-dire le « conservatisme opposé au changement $»$.

Le traitement de l'analyse factorielle des correspondances que nous avons appliqué à ces mêmes données et dont les résultats sont repris aux figures 12 et 13 mènent aux constatations suivantes. Les 3 premiers axes restituent $73 \%$ de la variance totale avec respectivement $34,8 \%, 21,5 \%$ et $16,8 \%$ pour chacun d'eux. Mise à part la transposition des deux premiers axes résultant du type différent d'analyse factorielle utilisé, on retrouve bien, représenté par le premier facteur (fig. 12), l'axe sémantique constitué par Frognier et Delfosse avec, à gauche, les socio-chrétiens, partisans du conservatisme, et, à l'extrême droite, les socialistes et les communistes, partisans d'un changement radical de notre société. Les autres partis occupent une position intermédiaire due principalement à leur attachement aux règles de l'économie libérale (ou mixte). Sur la figure 12 , on distingue nettement chacun des clivages caractéristiques de notre société politique. 


\section{Clivage socio-Economique}

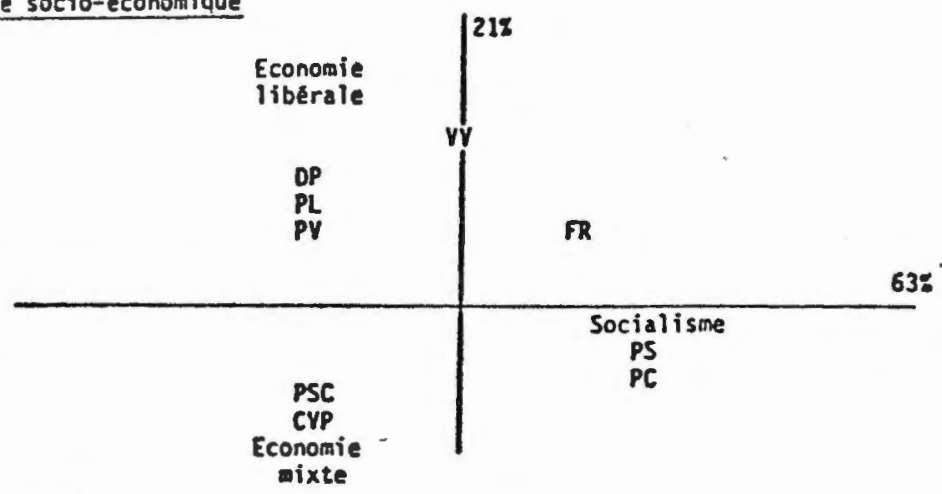

\section{Clivage moral}
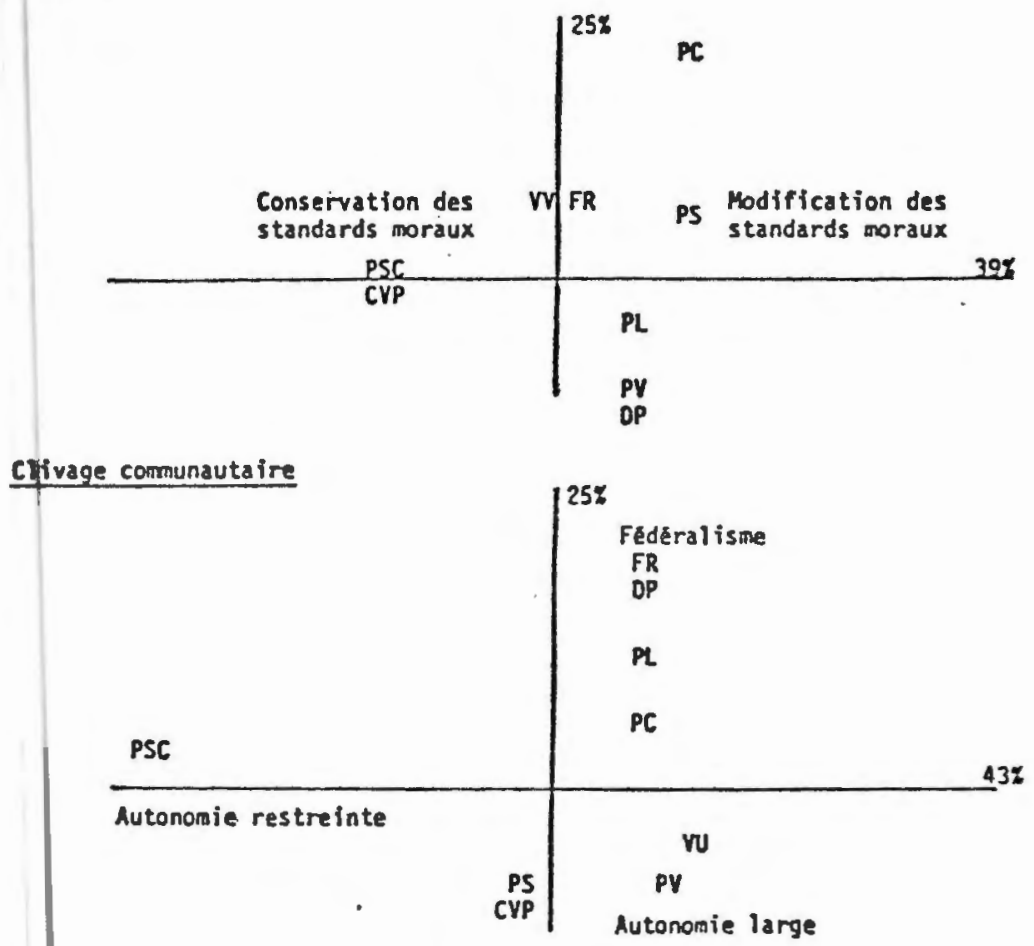

TIG 11. - Programmes 1974. Représentation graphique des résultats. 
Le second axe oppose les partis ayant les positions les plus extrêmes le long des axes sémantiques aux partis qui s'y caractérisent par une plus grande mobilité (les libétaux et les partis communautaires).

Observant que le positionnement des partis le long de cet axe est sensiblement identique à celui obtenu par Frognier et Delfosse, nous ne pouvons cependant les suivre lorsqu'ils considèrent cet axe comme une illustration $\mathrm{du}$ « catactère transidéologique de certains partis belges ». La caractéristique d'hétérogénéité idéologique que leur analyse attribue à certains partis résulte essentiellement de la manière dont a été appliqué le traitement statistique. Lorsque celui-ci opère indistinctement sur l'ensemble des observations positionnées selon les axes sémantiques prédéfinis, le résultat dépendra de la manière dont auront été associées les différentes définitions. Une analyse distincte portant sur les observations relatives à chacun des axes sémantiques peut mettre en lumière une hétérogénéité relative de certain (s) parti(s) et, ce, par rapport à l'un ou l'autre des axes sémantiques constitués. Une vue synthétique des résultats des analyses portant sur les différents thèmes semble indiquer que les partis communautaires affichent le caractère trans-idéologique le plus marqué bien que la position la plus extrême le long de ce second axe soit occupée par les partis libéraux. Quant au troisième axe, il dénote le particularisme des attitudes adoptées par les partis communautaires qui les distinguent des libéraux, notamment pour les problèmes éthiques, la détermination des limites de Bruxelles et leur conception en matière d'énergie. Il peut être intéressant d'observer que la conjonction des trois premiers axes s'inscrit dans un espace à trois dimensions et que leur positionnement dans cet espace forme un tétraèdre dont chacun des sommets localise une famille politique distincte. Enfin, le quatrième axe (12\%), non illustré ici, montre les divergences d'opinion entre les partis fédéralistes et semble bien illustrer l'observation de Rowies tappelée plus haut (point 2 ).

\subsection{Evolution des attitudes entre 1974 et 1978.}

Il nous reste à tirer les conclusions que l'analyse comparative des deux groupes de résultats, pour 1974 et pour 1978, met en évidence. Soulignons d'emblée que les différences de contenu sur lesquels ont porté les analyses incitent aux réserves d'usage quant à la portée et à Ia signification rigoureuse de pareille comparaison. Mais nous avons, malgré cette difficulté, estimé néanmoins intéressant de relever les éventuelles modifications d'attitudes.

Examinant dans un premier temps, l'évolution des associativités, nous observons qu'en 1974, selon les graphes synthétiques des associativités positives les plus fortes, l'ensemble des principaux partis se divise en trois blocs correspondant aux grandes familles politiques. Quatre ans plus tard, 


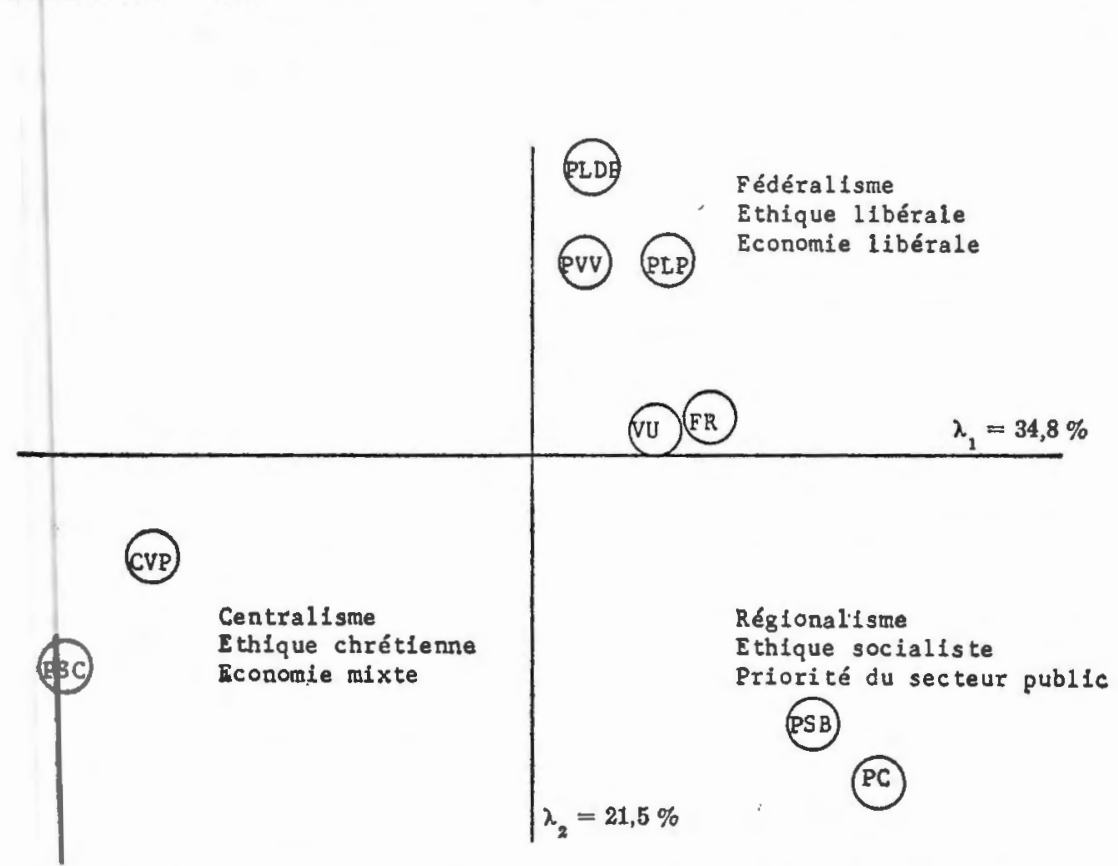

FIG. 12. - Programmes 1974. Représentation des axes factoriels 1 et 2.

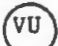

Pluralisme mitigé en matière scolaire

(ER)

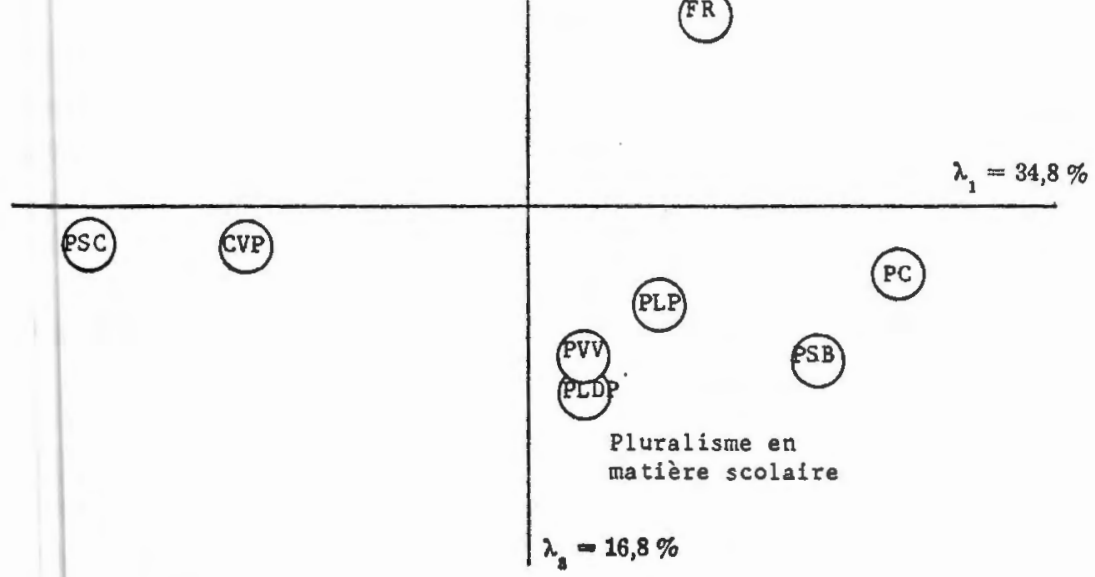

FIG. 13. - Programmes 1974. Représentation des axes factoriels 1 et 3. 
c'est le lien communautaire qui ressort nettement des associativités positives prédominantes, tout en révélant la plus grande homogénéité entre les partis néerlandophones en regard de la distinction plus tranchée au sein des partis francophones entre un premier sous-groupe formé du PSC et du PL, d'une part, et, d'autre part, un second réunissant le PS et le RW.

L'observation des associativités les plus négatives montrent que l'antagonisme entre familles chrétienne et socialiste s'est transformé entre 1974 et 1978 en une opposition entre partis régionalement dominants (PS en Wallonie et CVP en Flandre). Deux constatations intéressantes peuvent conclure ce paragraphe. La première est qu'il apparaît que le parti fédéraliste wallon a, depuis 1974, plus nettement opté pour un choix de société qui l'oppose tant aux chrétiens qu'aux libéraux et tout particulièrement aux thèses défendues par le PVV. La seconde veut mettre en évidence le chemin parcouru dans les esprits au sein du PSC qui semblent manifester une attitude assez restrictive quant au degré d'autonomie à reconnaître aux régions en 1974 et qui a assoupli considérablement sa position en la matière à la veille des dernières élections nationales.

$\mathrm{Si}$, ensuite, nous nous essayons à relever les modifications d'attitudes entre 1974 et 1978 telles qu'elles peuvent être dégagées des graphes synthétisant la position des partis à l'égard des différents thèmes, il peut être plus éclairant de distinguer ceux-ci selon qu'il s'agit de matières institutionnelles ou socio-économiques.

Alors que la conquête de l'autonomie régionale, en opposition aux téflexes unitaristes, constituait la dominante révélée par l'analyse des programmes des partis pour 1974, c'est plus nettement le clivage linguistique qui émerge en 1978. Une opposition d'attitudes (conservatisme opposé au changement) a laissé la place, nous semble-t-il, à une opposition de mentalités (une identité flamande opposée à une solidarité francophone). L'émergence d'une attitude propre aux partis régionalement dominants est également un phénomène nouveau en 1978. Par exemple, en ce qui concerne les compétences régionales ces partis dominants montrent plus d'exigence que leurs concurrents minoritaires.

Le clivage socio-économique, qui, en 1974, départageait nettement les partisans de l'interventionnisme public dans la vie économique des adeptes de l'économie libérale persiste en 1978. Mais, cette fois, les partis communautaires ont été amenés à se définir par rapport à cet axe, alors qu'en 1974 ils s'étaient peu démarqués. De plus, les résultats de 1978 révèlent un phénomène que le contenu de l'analyse de 1974 ne prenait pas en compte : la distance assez nette du groupe des « dogmatiques » rassemblant des partis installés dans l'opposition, par rapport au groupe des « pragmatiques » composé des partis de gouvernement. 
A noter, pour terminer, que les quatrièmes axes dans les deux séries d'analyses illustrent chacun une divergence orthogonale à un clivage-type tributaire du contenu respectif des deux analyses. En 1974, c'est la divergence d'opinion entre les " fédéralistes 》 qui se manifeste, tandis qu'en 1978, c'est au sein des « interventionnistes » que ce phénomène est perceptible.

\section{Conclusions.}

Les résultats d'une analyse factorielle appliquée au contenu des programmes politiques défendus en 1974 et 1978 nous ont permis d'établir les attitudes politiques caractérisant les principaux partis politiques belges et, dans une certaine limite, d'observer leur évolution dans le temps. La valeur informative d'une telle analyse est conditionnée par la qualité des informations originales. De meilleurs résultats pourraient être obtenus à partir d'un questionnaire établi de manière systématique quant au balayage du champ d'investigation. Ceci limiterait la marge d'appréciation arbitraire lors du codage des données. D'autre part, l'application répétée d'un tel questionnaire permettrait une évaluation beaucoup plus correcte de l'évolution des attitudes des partis. L'information qui en serait dégagée serait des plus utiles pour permettre à l'opinion publique de percevoir et d'apprécier plus correctement l'évolution des idées et des programmes en matìre politique.

Le positionnement différencié des différents partis ainsi que le déplacement de celui-ci, selon le type de thème analysé, semble bien révéler, de la part du parti, un comportement assez voisin de celui d'un entrepreneur cherchant à se placer le plus adéquatement possible par rapport à son marché (électorat) et par rapport à la concurrence (les autres partis) afin de maximiser son «profit » exprimé dans ce cas en termes de suffrages obtenus. Dans cet ordre d'idées, il serait également intéressant, d'une part, d'analyser plus spécifiquement les desiderata des électeurs - " consommateurs de produits politiques " - et, d'autre part, de procéder à l'égard de chacun de ces entrepreneurs, à une évaluation comparative de ses intentions (contenu de son programme) et de ses réalisations.

Si, a l'heure actuelle, il semble que ce sont plutôt les positions des électeurs (et leur taux de fidélité) qui suscitent l'intérêt des agents politiques, on peut penser que tout ce qui concourrait à une information plus rigoureuse et plus complète des mobiles déterminant l'action politique contribuerait au renforcement d'une société démocratique. 


\section{ANNEXE I}

Thèmes majeurs faisant l'objet des questions posées aux

présidents des principaux partis politiques belges (1978)

Thème

1. Réalisation des réformes Institutionnelles

2. Le - Pacte d'Egmont .

Compétences des ré. gions
Contenu de la question

- faít gravement défaut

- à peine de rupture de l'Etat

- dans des délais raisonnables

- urgence

- dans l'intérêt du pays

- dans l'intérêt de la région

- dans l'intérêt de la communauté

- le statu quo est préférable

- intervention indispensable des partis régionaux pour aboutir à une solution

- interdépendance entre problèmes socio-économiques et problèmes institutionnels

— faut-il une majorité des deux-tiers

- issue des négociations post-́́lectorales conditionnée par la personnalité du formateur

- constitue une base de négociation acceptable

- peut être repris tel quel

- accord communautaire préalabie à la constitution d'un gouvernement

- décentralisation des compétences nécessaire

- compétences plus étendues que celles prévues par le Pacte

- spécialement en matière de crédit ot fiscalité

- spécialement en matière d'enseignement et d'éducation

- spécialement en matlère d'environnement et d'aménagement du territoire

- spécialement pour les matlères personnalisables

- spécialement en matières de ressources naturelles [eau, forêt)

- autonomie complète vis à-vis du pouvolr central concernant ces compétences

- conflits de compétence réglés par une cour d'arbitrage

- résoudre ces conflits par voie de négociation

4. Compétences des communautes

- domalns des affalres pereonnallembles suscep-

\section{Thème}

5. Protection légale des droits des minorités

lingulstiques

Répartition du pouvolr décisionnel

7. Le problème bruxellols
Contenu de la question

- Ie jeu de la démocratie impilque-t-il que ia décision de la majorité doit prévaloir

- prévoir des garanties pour les minorités à Bruxelles

- prévoir des garanties pour les minorités dans la périphérie bruxelloise

- ces deux types de problèmes sont-ils comparables?

- pour le principe de la parité au niveau national

- pour le principe identique au niveau de Bruxelles (plutôt que droit de veto accord́́ aux minoritês)

- pour un système spécial de représentation au niveau national

- pour un système identique au niveau bruxellols

- pour une représentation proportionnelle assortie d'un droit de veto au niveau national

- pour une représentation de même nature au niveau bruxellois

- faut-il considérer qu'il y a trols régions

- accorder a Bruxelles un statut égal à colui des autres réglons

- possibilité d'une coalition des régions wallonne et bruxelloise

- limites des régions politiques calquées sur celles fixées par les lois linguistiques

- révision de la délimitation de la zone linguistique de la région bruxelloise

- les populations concernées devraient être consultées

- partage de la périphérie bruxelloise selon l'homogénéité linguistlque

- zone économique bruxellolse Implique-telle reconnaissance d'une lialson entre la périphérie et la zone urbaine bruxellolse. tible d'extension

- cala constltue-t-il une gerantie suffisante contre la minorisation 


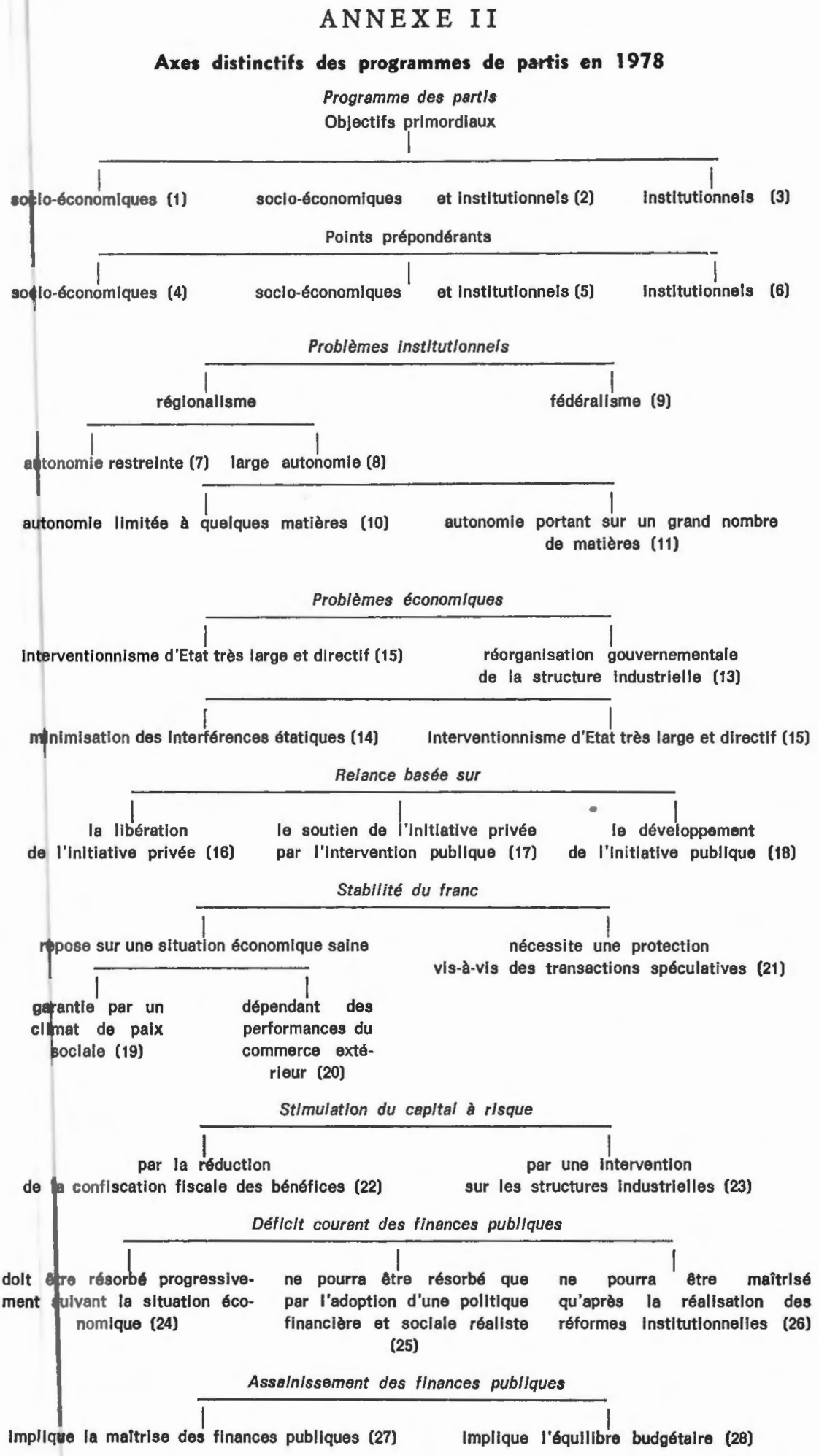


ANNEXE I I I

Types de codages testés et leur répartition dans le tableau des données

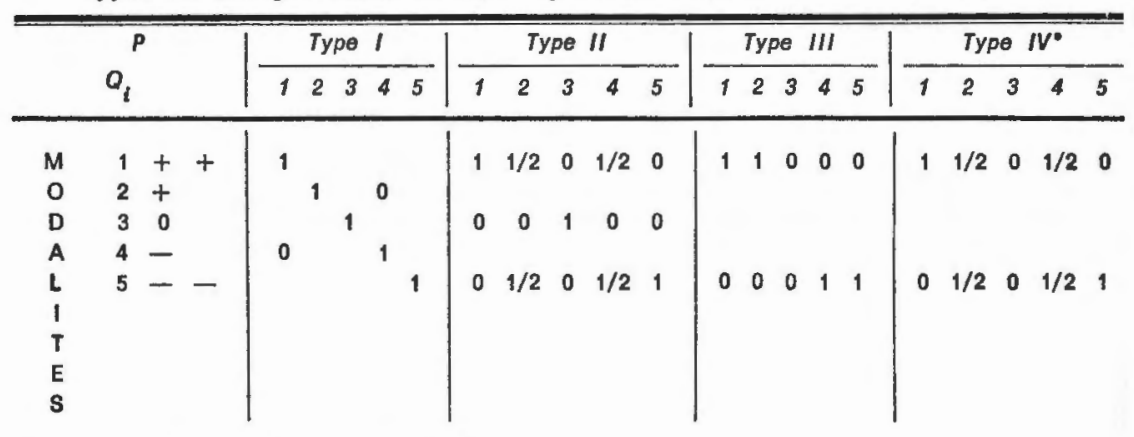

- Codage retenu pour la présente étude.

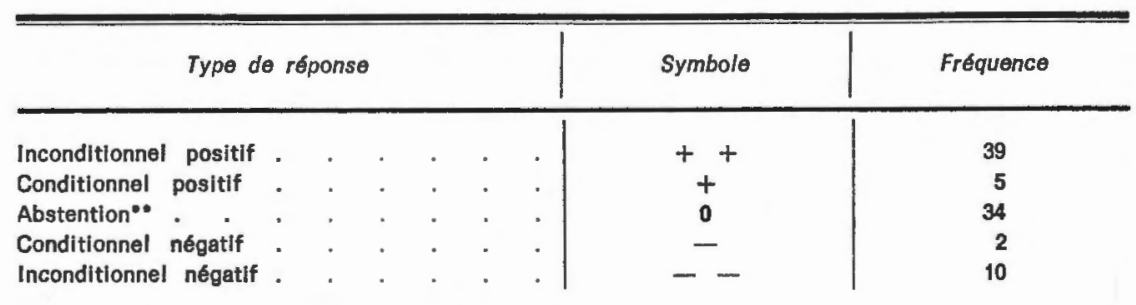

- Y assimilées les réponses floues et ambiguēs.

\section{Summary : A tentative visualisation of the major Belgian political parties attitudes.}

The main point developed in this paper is the demonstration of the attitudes characterizing the major Belgian political parties. This is achieved through a factorial analysis applied to a set of data resulting from the answers given by the major parties leaders to various specific questions regarding both the prevailing economic as well as institutional issues at the eve of the election of December 17th, 1978. From that point it has been possible to compare those results with the one obtained from tbe data concerning the 1974 programmes.

The results of the statistical analysis show that while in 1974 the strongest positive associations between parties corresponded to the traditional etbical and socioeconomic cleavages, four years later the linguistic dimension was predominant with a stronger bomogeneity characterizing the Dutchspeaking group than what could be observed for the Frenchspeaking one.

The factorial analysis allows for a graphical representation of the respective positioning of the parties along the statistically significant factorial axes. Applied to two sets of data, the first one on purely institutional matters, the second one on both institutional and economic policies, the analysis reveals clearly through the various graphs presented the linguistic cleavage as well as the divergences about the extent of regional competences and minorities protection, on the one hand, and, on the other band, the degrees of regional economic autonomy and of public intervention in the business life.

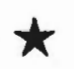

